\title{
Frontoparietal Structural Connectivity in Childhood Predicts Development of Functional Connectivity and Reasoning Ability: A Large-Scale Longitudinal Investigation
}

\author{
Carter Wendelken, ${ }^{1,2}$ Emilio Ferrer, ${ }^{3}{ }^{\circledR S}$ Simona Ghetti, ${ }^{3}{ }^{\circledR}$ Stephen K. Bailey, ${ }^{4}$ Laurie Cutting, ${ }^{4}$ and ${ }^{\circledR S i l v i a ~ A . ~ B u n g e ~}{ }^{1}$ \\ ${ }^{1}$ Helen Wills Neuroscience Institute and Department of Psychology, University of California, Berkeley, California 94720, ${ }^{2}$ Vicarious FPC, Inc., Union City, \\ California 94587, ${ }^{3}$ Department of Psychology and Center for Mind and Brain, University of California, Davis, California 95616, and ${ }^{4}$ Peabody College of \\ Education and Human Development, Vanderbilt University, Nashville, Tennessee 37240
}

Prior research points to a positive concurrent relationship between reasoning ability and both frontoparietal structural connectivity (SC) as measured by diffusion tensor imaging (Tamnes et al., 2010) and frontoparietal functional connectivity (FC) as measured by fMRI (Cocchi et al., 2014). Further, recent research demonstrates a link between reasoning ability and FC of two brain regions in particular: rostrolateral prefrontal cortex (RLPFC) and the inferior parietal lobe (IPL) (Wendelken et al., 2016). Here, we sought to investigate the concurrent and dynamic, lead-lag relationships among frontoparietal SC, FC, and reasoning ability in humans. To this end, we combined three longitudinal developmental datasets with behavioral and neuroimaging data from 523 male and female participants between 6 and 22 years of age. Cross-sectionally, reasoning ability was most strongly related to FC between RLPFC and IPL in adolescents and adults, but to frontoparietal SC in children. Longitudinal analysis revealed that RLPFC-IPL SC, but not FC, was a positive predictor of future changes in reasoning ability. Moreover, we found that RLPFC-IPL SC at one time point positively predicted future changes in RLPFC-IPL FC, whereas, in contrast, FC did not predict future changes in SC. Our results demonstrate the importance of strong white matter connectivity between RLPFC and IPL during middle childhood for the subsequent development of both robust FC and good reasoning ability.

Key words: fMRI; functional connectivity; longitudinal; reasoning; structural connectivity

Significance Statement

The human capacity for reasoning develops substantially during childhood and has a profound impact on achievement in school and in cognitively challenging careers. Reasoning ability depends on communication between lateral prefrontal and parietal cortices. Therefore, to understand how this capacity develops, we examined the dynamic relationships over time among white matter tracts connecting frontoparietal cortices (i.e., structural connectivity, SC), coordinated frontoparietal activation (functional connectivity, FC), and reasoning ability in a large longitudinal sample of subjects 6-22 years of age. We found that greater frontoparietal SC in childhood predicts future increases in both FC and reasoning ability, demonstrating the importance of white matter development during childhood for subsequent brain and cognitive functioning.

\section{Introduction}

Reasoning, or the capacity to solve problems in novel situations, is a form of high-level cognition that improves dramatically over

Received Dec. 4, 2016; revised June 9, 2017; accepted June 20, 2017.

Author contributions: C.W., E.F., S.G., L.C., and S.A.B. designed research; C.W. and S.K.B. performed research; C.W. analyzed data; C.W., S.G., and S.A.B. wrote the paper.

This work was supported by the National Science Foundation (Grant BCS1558585). Funding for the original data collection was provided by the National Institutes of Health (National Institute of Neurological Disorders and Stroke Grant R01 NS057156, National Institute of Mental Health Grant R01 MH091109, and National Institute of Child Health and Human Development Grant R01 HD067254). S.A.B.'s contribution was additionally supported by a Jacobs Foundation Research Fellowship. We thank former and current members of the Bunge, Ghetti, and Cutting laboratories for assistance with data collection and Yana Fandakova and Susan Whitfield-Gabrieli for feedback on the manuscript.

The authors declare no competing financial interests. childhood and, to a lesser extent, during adolescence (McArdle et al., 2002; Ferrer et al., 2009). Much of the research on the neural underpinning of reasoning has focused on its localization to specific brain regions (Prado et al., 2011; Wendelken et al., 2011; Krawczyk, 2012). However, reasoning, like other higher cognitive operations, depends on the coordinated action of multiple regions. Therefore, characterizing patterns of interregional communication and how such communication changes over time is

Correspondence should be addressed to either Carter Wendelken or Silvia A. Bunge, Helen Wills Neuroscience Institute and Department of Psychology, 174 Li Ka Shing Center, MC\#3370, University of California, Berkeley, CA 94720. E-mail: cwendelken@gmail.com or sbunge@berkeley.edu.

DOI:10.1523/JNEUROSCI.3726-16.2017

Copyright $\odot 2017$ the authors $\quad 0270-6474 / 17 / 378549-10 \$ 15.00 / 0$ 
critical for understanding developmental changes and individual differences in reasoning. Doing so requires insight into both structural connectivity (SC), the white matter tracts that connect disparate brain regions, and functional connectivity (FC), the coordinated activity of different regions.

Considerable effort has been devoted to understanding the development of FC and how it relates to higher cognition. In particular, prior research has emphasized the importance of FC among components of the lateral frontoparietal network (LFPN), which supports reasoning and other higher cognitive functions (Jung and Haier, 2007; Shokri-Kojiri et al., 2012; Cocchi et al., 2014). Prior studies have also examined links between SC and reasoning, reporting associations between reasoning and multiple white matter tracts (Tamnes et al., 2010; Peters et al., 2014) or between reasoning and global white matter during childhood (Ferrer et al., 2013).

Our prior fMRI research in adults has emphasized the importance for reasoning of two brain regions in particular: rostrolateral prefrontal cortex (RLPFC) and the inferior parietal lobule (IPL) (Wendelken et al., 2010, 2011, 2012; Krawczyk, 2012; for review, see Vendetti and Bunge, 2014). In a cross-sectional fMRI study that focused on FC among key nodes of the LFPN, we demonstrated that RLPFC-IPL FC and a composite measure of reasoning ability were associated in adolescents, but not in children under 12 (Wendelken et al., 2016). The present study examines concurrent and longitudinal predictors of reasoning ability in a large, pooled dataset that includes the earlier sample.

To understand how LFPN FC emerges as a contributor to reasoning ability, it is critical to also understand the relevant changes in SC and how these relate to changes in FC and reasoning ability. Prior work relating SC and FC has focused on the default mode network (Greicius et al., 2009; Horn et al., 2014; Khalsa et al., 2014) or on global patterns of connectivity (Honey et al., 2009), with little attention given to the LFPN connections that are critical for higher cognition. Moreover, whereas concurrent relationships between SC and FC have been explored for some networks, the dynamic, lead-lag relationships between these measures remain largely uncharted.

Here, we considered two nonmutually exclusive hypotheses about the lead-lag relations between SC and FC in the LFPN. First, SC could enable the development of FC such that increased $\mathrm{SC}$ would be associated with greater potential for future increases in FC. Second, functional interaction between regions could drive physiological changes in the white matter connections. In this case, higher FC would be associated with future increases in SC. We then sought to determine whether SC and/or FC relate to reasoning development. It is possible that any relationship between SC and behavior, whether concurrent or lagged, is mediated by FC. Alternatively, even where there is a concurrent relationship between FC and behavior, SC may still prove to be a better predictor of behavioral change. In this case, earlier maturation of white matter tracts may be associated with earlier improvements in reasoning performance. However, delayed maturation might be advantageous (Shaw et al., 2006). The present study tests these hypotheses regarding the lead-lag relationships among SC, FC, and reasoning ability over development.

\section{Materials and Methods}

Experimental design. To begin to answer these questions about the leadlag relationships among SC, FC, and reasoning ability, we conducted an analysis of longitudinal diffusion tensor imaging (DTI), fMRI, and behavioral data from $>520$ participants enrolled in longitudinal brain imaging research. We focused our analyses on the LFPN connections that
Table 1. Number of participants with data for each measure from each contributing dataset

\begin{tabular}{|c|c|c|c|c|c|c|c|c|c|c|}
\hline \multirow[b]{2}{*}{ Study } & \multirow[b]{2}{*}{ Age range } & \multicolumn{3}{|c|}{ Matrix reasoning } & \multicolumn{3}{|l|}{ DTI } & \multicolumn{3}{|l|}{ fMRI } \\
\hline & & $N_{\mathrm{p}}$ & $N_{1}$ & $N_{\mathrm{t}}$ & $N_{\mathrm{p}}$ & $N_{\mathrm{I}}$ & $N_{\mathrm{t}}$ & $N_{\mathrm{p}}$ & $N_{\mathrm{I}}$ & $N_{\mathrm{t}}$ \\
\hline NORA & $6-20$ & 191 & 118 & 311 & 137 & 48 & 187 & 141 & 54 & 198 \\
\hline HIPPO & $7-15,18-21$ & 211 & 105 & 314 & 181 & 131 & 392 & 164 & 97 & 322 \\
\hline LERD & $7-11$ & 119 & 0 & 119 & 116 & 78 & 244 & 91 & 9 & 100 \\
\hline
\end{tabular}

$N_{p}$, number of unique participants; $N_{1}$, number of participants with longitudinal data; $N_{t}$, total number of participant visits.

have been implicated previously in studies of reasoning, and in particular on the RLPFC-IPL connection that our own prior research has highlighted as a key contributor to reasoning ability. The present study extends this prior work by examining how FC among frontal and parietal ROIs relates to SC and by examining longitudinal relationships among these brain variables and behavior.

Participants and measures. This study incorporated data from three different sources: (1) the Neurodevelopment of Reasoning Ability study (NORA; UC Berkeley, PIs: S.A.B. and E.F.), (2) the Hippocampal Investigation of Pediatric Populations over Time study (HippoTime; UC Davis; PIs: S.G. and S.A.B.), and (3) the Predicting Late-Emerging Reading Disability study (LERD; Vanderbilt University; PI: L.C.). Altogether, the current study included data from 523 participants (254 females), including 193 from the NORA dataset, 211 from the HippoTime (HIPPO) dataset, and 119 from the LERD dataset. Longitudinal data from two (NORA), three (HIPPO), or four (LERD) time points were available for 345 of these participants. Participants ranged in age from 6 up to 21.7 years, with a mean age of 10.74 years $(S D=3.29$ years). A more detailed breakdown of participant demographics is given in Table 1.

The NORA and HIPPO studies both involved a cohort-sequential longitudinal design wherein $\mathrm{T} 1$ samples were collected from a broad initial age range and follow-up scans were collected at different intervals. For NORA, T2 data collection was conducted on average 1.5 years after T1 data collection (ranging from $0.9-2.2$ years). For HIPPO, longitudinal visits were separated by an average of 1.3 years (ranging from $0.73-2.9$ years). LERD used a traditional longitudinal design in which all T1 data were collected from participants at age 7 and subsequently at 1-year intervals.

We considered three principal measures for each participant: (1) reasoning ability, indexed via raw scores from the WASI Matrix Reasoning Test (Wechsler, 1974); (2) SC, indicated by fractional anisotropy (FA) of selected white-matter tracts; and (3) FC, calculated as task-independent interregional correlations in fMRI time series data. SC data were available for all time points in all three studies. However, HIPPO participants had matrix reasoning scores from only $\mathrm{T} 1$ and T3, LERD participants had matrix reasoning scores only at $\mathrm{T} 1$, and FC only at $\mathrm{T} 1$ and $\mathrm{T} 4$. Although the LERD dataset included resting-state fMRI data, both NORA and HIPPO contributed task data. NORA fMRI data were collected while subjects performed a visual analogy task (Whitaker et al., unpublished data; task described in Wright et al., 2007). HIPPO fMRI data were collected while subjects performed a source memory task (Sastre et al., 2016).

In our prior investigations of reasoning ability with the NORA dataset, we computed a reasoning ability factor score on the basis of multiple reasoning measures, including concept formation and analysis-synthesis from Woodcock-Johnson III (Woodcock et al., 2001) as well as block design and matrix reasoning from WASI (Ferrer et al., 2013; Wendelken et al., 2016). Matrix Reasoning, the only measure that was available across the three studies that we consider here, loaded strongly onto the reasoning factor score in NORA in previous analyses (Ferrer et al., 2013).

MRI data collection and preprocessing. NORA data were collected at the University of California at Berkeley Brain Imaging Center and the University of California at San Francisco Neuroimaging Center on $3 \mathrm{~T}$ Siemens TIM MR scanners with 12-channel head coils. HIPPO data were collected at the UC Davis Imaging Research Center on a 3 T Siemens Trio Tim scanner with a 32-channel head coil. LERD data were collected at Vanderbilt University Institute of Imaging Science on a $3 \mathrm{~T}$ Philips Achieva MRS scanner. Details of each scan type are included in Table 2. 
Table 2. Scan parameters for the DTI and fMRI scans for each contributing dataset

\begin{tabular}{|c|c|c|}
\hline Study & DTI & fMRI \\
\hline \multirow[t]{4}{*}{ NORA } & One $9.45 \mathrm{~min}$ EPI scan & Four $4.06 \mathrm{~min}$ EPI scans \\
\hline & $64 \mathrm{dir}, \mathrm{TR}=7900 \mathrm{~ms}, \mathrm{TE}=102 \mathrm{~ms}$ & $\mathrm{TR}=2000 \mathrm{~ms}, \mathrm{TE}=25 \mathrm{~ms}$ \\
\hline & $2.2 \mathrm{~mm}^{3}$ isotropic voxels & 33 slices, $2.0 \times 1.8 \times 3.0 \mathrm{~mm}^{3}$ voxels \\
\hline & $B_{1}=0, B_{2}=2000 \mathrm{~s} / \mathrm{mm}^{2}$ & Visual analogy task \\
\hline \multirow[t]{4}{*}{ HIPPO } & One 8.32 min EPI scan & Six EPI scans (3@ @ min \& $3 @ 6.3 \mathrm{~min})$ \\
\hline & $64 \mathrm{dir}, \mathrm{TR}=7400 \mathrm{~ms}$, TE $=81 \mathrm{~ms}$ & $\mathrm{TR}=2000 \mathrm{~ms}, \mathrm{TE}=23 \mathrm{~ms}$ \\
\hline & $2.5 \times 2.2 \times 2.2 \mathrm{~mm}^{3}$ voxels & 37 slices, $3 \mathrm{~mm}^{3}$ isotropic voxels \\
\hline & $B_{1}=0, B_{2}=1000 \mathrm{~s} / \mathrm{mm}^{2}$ & Source memory task \\
\hline \multirow[t]{4}{*}{ LERD } & One 9.32 min EPI scan (HARDI) & One 5.87 or $7.32 \mathrm{~min}$ EPI scan (T1/T4) \\
\hline & $60 \mathrm{dir}, \mathrm{TR}=8600 \mathrm{~ms}$, TE $=66 \mathrm{~ms}$ & $\mathrm{TR}=2200 \mathrm{~ms}, \mathrm{TE}=30 \mathrm{~ms}$ \\
\hline & $2.5 \mathrm{~mm}^{3}$ isotropic voxels & 35 slices, $3 \mathrm{~mm}^{3}$ isotropic voxels \\
\hline & $B_{1}=0, B_{2}=2000 \mathrm{~s} / \mathrm{mm}^{2}$ & Resting-state \\
\hline
\end{tabular}

DTI data were analyzed using the FMRIB Diffusion Toolbox (FDT) software tool (Behrens et al., 2003). First, eddy correction was run on the DTI images to correct for eddy current distortions and brain extraction was performed to exclude nonbrain voxels from further analysis. After these preliminary steps, a DTI model was fit to each voxel to calculate directions and magnitude of diffusion. This procedure produces an FA image for each participant.

All fMRI data were preprocessed in SPM8 (Wellcome Trust Center for Neuroimaging, London). Functional images were corrected for differences in slice acquisition timing and were realigned to the first volume by means of a six-parameter rigid-body transformation. Each participant's T1 structural image was coregistered to his/her mean realigned functional image and then spatially normalized to SPM's T1 template. Normalization parameters obtained from this process were then applied to the functional images to produce a set of functional images in SPM standard space (MNI 152), with $3 \times 3 \times 3 \mathrm{~mm}$ voxels. Functional images were then smoothed with an $8 \mathrm{~mm}$ FWHM isotropic Gaussian kernel. Finally, volumes associated with a high degree of motion $(>1 \mathrm{~mm}$ scan-toscan translation) or signal spiking ( $>2 \%$ signal change) were corrected (interpolated) using the ArtRepair volume correction tool (Stanford Psychiatric Neuroimaging Laboratory). Scans with $>25 \%$ corrected volumes were excluded from further analysis, resulting in exclusion of 346 separate scans, or $11 \%$ of the total number available.

FC analysis. We sought to understand the relationships among reasoning ability, SC, and intrinsic patterns of FC, correlations in regional activation that are relatively stable across task demands and that are thought to reflect the long-term history of coordination between regions (Seeley et al., 2007; Cole et al., 2014). Therefore, we adopted the method of intrinsic FC analysis. This approach contrasts with analyses of taskrelated FC that focus on higher-frequency correlations that differ as a function of task demands. Although our pooled dataset included data from several fMRI scans (NORA and HIPPO) in addition to resting-state scans (LERD), our analysis was designed to minimize the effect of task on the connectivity measure.

ROIs were $5 \mathrm{~mm}$ spheres that we have used previously in the examination of FC and reasoning (Wendelken et al., 2016; Fig. 1). Each ROI was centered on coordinates selected from a large set of ROIs that have been used previously to examine global connectivity properties (Power et al., 2011). Specifically, we selected coordinates that corresponded to the left and right RLPFC, DLPFC, IPL, and SPL regions that are typically engaged during reasoning tasks (Krawczyk, 2012; Vendetti et al., 2014).

Intrinsic FC between these regions was assessed by measuring lowfrequency correlations between BOLD activation time series extracted for each ROI and for each participant. Several steps were undertaken to minimize the effects of physiological noise and motion on the extracted time series: (1) regressing out average signal from CSF and from white matter; (2) regressing out volume-to-volume motion for six dimensions (three translation directions and three rotation axes); (3) regressing out task-related signal where applicable (Fair et al., 2007); (4) band-pass filtering (with a minimum frequency of $.008 \mathrm{~Hz}$ and a maximum frequency of $0.09 \mathrm{~Hz}$ ); and (5) scrubbing (Power et al., 2012) to remove time points associated with high motion and/or signal spikes (as determined by ArtRepair). Correlation values were transformed to $Z$-scores using Fisher's $R$-to- $Z$ transformation before the main statistical analyses.

$S C$ analysis. White matter tracts were obtained via probabilistic tractography, using the FDT ProbtrackX tool. Tractography was conducted using T1 data from the NORA dataset and these tracts were subsequently used for the analysis across all longitudinal time points and datasets. The same lateral prefrontal and parietal coordinates that were used in analyses of FC (as centers for the spherical ROIs) were also used as end points for tractography. Computed tracts included left and right frontoparietal, intrafrontal, and intraparietal connections (Fig. 2). For each participant and each target tract, 1000 attempts were made to find a streamline from one end point to the other. Specifically, each streamline was started randomly from a white matter voxel within a $12 \mathrm{~mm}$ radius of the start point and terminated successfully if it reached a voxel within $12 \mathrm{~mm}$ of the end point (the $12 \mathrm{~mm}$ radius was selected to ensure sufficient white matter voxels within the sphere). A voxel was considered to be part of a subject's tract if at least two streamlines passed through that voxel. Group average tracts were obtained by registering binarized subject tracts to MNI space, summing these together, and then thresholding to include in the final group tract voxels that were present in at least $25 \%$ of the contributing subject tracts. For each participant, the group tracts were mapped to subject space and masked with segmented subject white matter to produce subject-specific versions of each tract. For the resulting masks, average FA values were extracted from the participant's FA map and these FA values were submitted to statistical analyses.

Statistical analysis. All statistical analyses were conducted in R (R Core Team, 2013). To account for possible differences between the three datasets (e.g., due to scanner, testing conditions), all values were normalized to the NORA sample. Specifically, for each measure from the LERD or HIPPO dataset (e.g., left RLPFC-IPL fractional anisotropy), a mean value was calculated both for the LERD/HIPPO sample and for the equivalent age range from the NORA sample. The difference was then subtracted from the LERD/HIPPO sample values to produce a set of normalized values for that measure. For HIPPO matrix reasoning, due to the fact that different test versions were used at the two different time points, data from each time point were normalized separately to the relevant NORA sample mean. In addition to normalization, we also included study (NORA, HIPPO, or LERD) as a covariate of no interest in relevant analyses (as noted).

We consider the combination of datasets, as described above, to be the best approach given the available data. First, although our full participant sample is large, no individual dataset contributed sufficient numbers of data points to fully support our planned longitudinal analyses. Second, individual datasets included narrower age ranges and/or gaps in coverage relative to the combined dataset. Nevertheless, our approach may not fully succeed at controlling for differences between datasets and there is added value in also examining results from the separate datasets. Therefore, for each of the main results that we present, we also report corresponding results from each individual dataset.

Before normalization, we conducted outlier correction separately for each dataset and relevant measure. We removed data points that were $>3$ SDs from the mean for that dataset. In addition, after normalization, we removed data points that were $>3$ SDs from the mean of the entire sample. In total, eight subject time points were excluded due to outlier matrix reasoning scores, another five due to outlier FC values, and another 10 due to outlier SC values.

Concurrent relationships and age effects were examined using mixed model regression, which accounts for subject repetition, on the full longitudinal dataset (nlme package in R; R package version 3.1-131, https:// CRAN.R-project.org/package $=$ nlme. Longitudinal analyses involved modeling and regression of change scores and were limited in all cases to data from two longitudinal time points.

To test for age differences in the relationships between variables, we split the full age range into two separate age groups. Having previously shown a developmental shift in FC that occurs at around age 12 (Wendelken et al., 2016), we created a subdivision between children under 12 (ages 6-11, here termed "younger participants") and adolescents and young adults (ages 12-22, termed "older participants"). This procedure 
resulted in 423 individuals (209 females) in the former group and 99 individuals (48 females) in the latter.

In addition to splitting the data by age group for a subset of analyses, we also examined nonlinear age-related changes in our key variables using age as a continuous variable. For our key measures, including matrix reasoning, RLPFC-IPL FC, and RLPFC-IPL SC, trajectories of age-related change, computed across the entire age range, were fit using the cumulative normal distribution ("pnorm" function in R). This function includes two parameters: $\mu$, the age of maximal change, and $\sigma$, the spread of change around that age. This model can fit a variety of different trajectories, including linear, asymptotic, and s-shaped curves, and has the advantage of doing so with a single functional form that yields readily interpretable parameters (Wendelken et al., 2016).

\section{Results}

Developmental improvement in reasoning ability

Reasoning ability, as indexed by scores on the matrix reasoning task, demonstrated robust developmental improvement. In crosssectional analysis of the full age range, we observed a nonlinear pattern of age-related increase, with the greatest increases occurring among the youngest participants $\left(b=33.4, t_{(311)}=27.6, p<\right.$ 0.001 ; Fig. 3 ). We found that age-related increases were maximal at age 6; that is, at the beginning of the examined age range. There was no effect of gender on behavioral performance. Although younger participants demonstrated the larger increase in reasoning ability, in both linear cross-sectional and longitudinal analyses (cross: $b=2.4, t_{(149)}=19.1, p<0.001$; longitudinal change in $\left.R: \Delta=3.97, t_{(201)}=11.2, p<0.001\right)$, older participants also showed significant improvement (cross: $b=0.53, t_{(60)}=5.9, p<$ 0.001 ; longitudinal change in $\left.R: \Delta=1.8, t_{(45)}=3.0, p=0.004\right)$.

\section{Test of potential confound for brain imaging analyses}

Recent research has suggested that many behavioral measures, including reasoning ability, might be related to in-scanner head motion (Siegel et al., 2016). Therefore, we sought to test for this possibility in our own data by regressing matrix reasoning score against average volume-to-volume translational displacement. Although increasing age was associated with decreasing head motion in our sample, as expected, the relationship between motion and reasoning ability was not significant after accounting for age $(p=0.34)$. Further, there was no interaction between motion and age group (younger vs older participants) in their effect on reasoning $(p=0.41)$. Despite the lack of a relation between head motion and reasoning ability in our sample of participants, as described previously, we used methods designed to reduce the impact of head motion on computed FC measures (regression of motion parameters and scrubbing).

\section{Connectivity and reasoning ability: concurrent effects}

Before addressing the key question of the lead-lag relationships among SC, FC, and reasoning, we sought to extend, in this larger sample, prior results obtained with the NORA dataset that were focused on the separate cross-sectional relationships between reasoning ability and FC (Wendelken et al., 2016) and between reasoning ability and SC (Ferrer et al., 2013). The former study included 132 participants (76 males, age 6-19), with T2 longitudinal data for 56 . The latter study included cross-sectional data from 103 participants (55 males, age 6-18). All of these participants were included in the present analysis.

As reported previously for a subset of the present dataset (Wendelken et al., 2016), there was an interaction between age group (6-11 vs 12-22) and RLPFC-IPL FC $\left(b=8.2, t_{(100)}=2.6\right.$, $p=0.009)$, with the FC-reasoning relation present in older participants (controlling for age and study: $b=5.44, t_{(18)}=3.1, p=$ $0.006)$, but not in younger participants $(p=0.24)$. The result in older participants was driven by a significant effect for left RLPFC-IPL $\left(b=4.2, t_{(19)}=2.8, p=0.01\right)$ and a nonsignificant trend for right RLPFC-IPL $\left(b=2.1, t_{(19)}=1.5, p=0.16\right)$. Separate analysis of older participants in the NORA and HIPPO datasets revealed similar positive effects: significant in the former and marginally significant in the latter (NORA: $b=8.2, p=0.002$; HIPPO: $b=$ $4.2, p=0.1)$. In contrast, there was no effect for younger participants in any of the three datasets examined separately (all $p>$ 0.2 ). There were no main effects or interactions involving gender $(p>0.2)$. In summary, we found a relationship between RLPFCIPL FC and reasoning only for adolescents and young adults.

Next, we examined LFPN FC more generally. However, we found no significant relationship between reasoning ability and either average frontoparietal connectivity (i.e., average across connectivity of all frontal to parietal connections) or average network connectivity (i.e., average across all frontoparietal, intraprefrontal, and intraparietal connections), in either younger or older participants $(p>0.21)$. Therefore, reasoning ability among adolescents and young adults was related to FC of RLPFC-IPL, particularly in the left hemisphere, but not to connectivity among other nodes in the LFPN.

In the next set of analyses, we focused on SC. Having shown in cross-sectional analyses of a subset of the NORA dataset that SC averaged across all white matter in the brain was positively related to reasoning ability (Ferrer et al., 2013), we tested here whether SC within the LFPN and/or for frontoparietal connections in particular would be related to reasoning ability. First, we computed average SC across all of our tracts: frontoparietal tracts as well as intrafrontal and intraparietal tracts. Mixed-model regression analyses conducted on the full sample revealed a marginally significant positive relation between average frontoparietal network SC and reasoning ability (controlling for age and study; $b=$ $\left.23.3, t_{(128)}=1.8, p=0.06\right)$.

To test whether this marginal relation was driven by a subset of connections within the network, we broke LFPN SC down into three separate components: frontoparietal SC, intrafrontal SC, and intraparietal SC. In a stepwise regression across the entire age range, only frontoparietal SC survived as a predictor of reasoning ability. This overall relationship between frontoparietal $\mathrm{SC}$ and reasoning was marginally significant when controlling for 


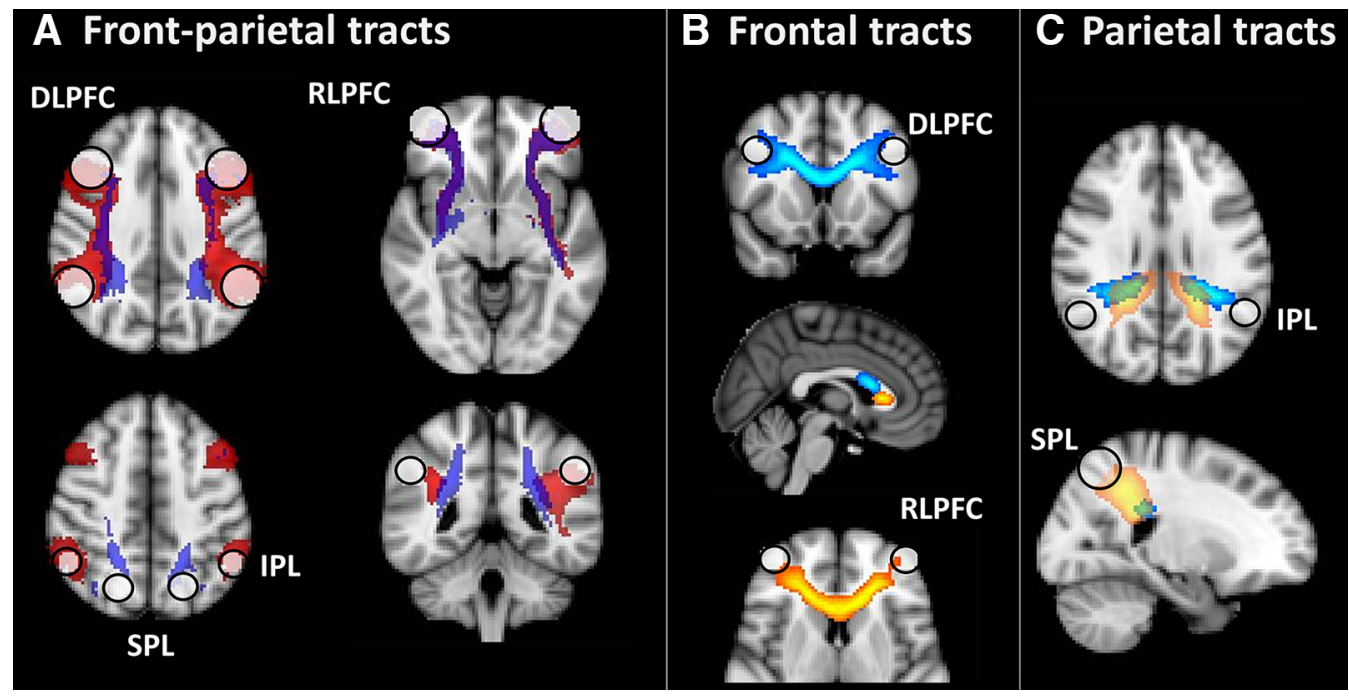

Figure 2. White matter tracts, obtained via probabilistic tractography, including frontoparietal tracts from RLPFC or DLPFC to IPL (red) or SPL (blue) (A); bilateral prefrontal tracts between left and right DLPFC (blue) and between left and right RLPFC (yellow) (B); and bilateral parietal tracts between left and right IPL (blue) and between left and right SPL (yellow) (C). End-point masks (12 mm spheres) are indicated with white circles. Purple $(\boldsymbol{A})$ and green $(\boldsymbol{B}, \boldsymbol{C})$ indicate overlap between tracts.

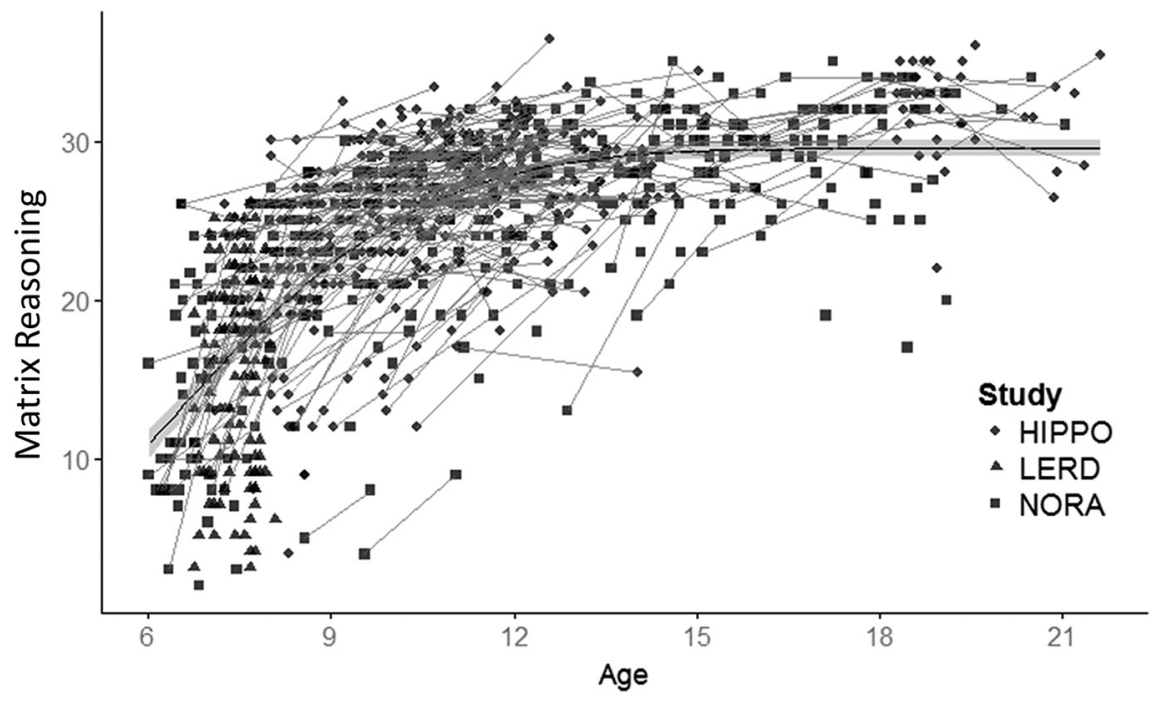

Figure 3. Scatter plot of the relationship between age and matrix reasoning. Gray lines indicate longitudinal data. The fit line was calculated using the cumulative distribution function (pnorm in R). Optimal parameters $\mu=6$ and $\sigma=3.5$, extracted from the data, indicate maximal change at age 6 (i.e., at the beginning of the examined age range).

both age and study $\left(b=26.1, t_{(127)}=1.9, p=0.06\right.$; Fig. 4$)$. There was no interaction between SC and gender $(p>0.2)$.

Next, we investigated whether this relationship between frontoparietal SC and reasoning varied as a function of age. We included an age $\times$ SC interaction term into the regression model and found a negative interaction between the effects of age and frontoparietal SC on reasoning $\left(b=-16.5, t_{(127)}=-5.2, p<\right.$ 0.001 ) such that there was a bigger effect of SC on reasoning among younger participants. Further, the main effect of SC on reasoning was significant in this model $\left(b=194.0, t_{(127)}=5.5\right.$, $p<0.001)$. A similar analysis that incorporated age as a categorical group rather than as continuous variable revealed a similar interaction (age group $\times \mathrm{SC}: b=-13.5, t_{(127)}=-3.8, p<$ $0.001)$, such that there was a strong positive relationship between frontoparietal SC and reasoning in younger participants $(b=$ $\left.88.7, t_{(64)}=5.4, p<0.001\right)$, but not in older participants $(p=$ $0.54)$. In summary, stronger SC within frontoparietal tracts was associated with better reasoning ability in children but not in adolescents and adults.

To better understand this key finding of a positive effect of frontoparietal SC on reasoning in younger participants, we repeated the previous age interaction analysis separately in each of the three datasets. The negative interaction between age and SC was observed in all three datasets (NORA: $b=$ $-21.8, t_{(44)}=-4.9, p<0.001$; HIPPO: $b=-9.7, t_{(81)}=-2.3, p=0.03$; LERD: $\left.b=-256.9, t_{(84)}=-1.7, p=0.09\right)$. Further, in the presence of this interaction, the positive main effect of SC on reasoning was also apparent in all three datasets (NORA: $b=251.0, t_{(44)}=5.2, p<0.001$; HIPPO: $b=120.4, t_{(81)}=2.3, p=0.03$; LERD: $b=$ $\left.1919.1, t_{(84)}=1.7, p=0.09\right)$.

Next, we sought to determine whether the association between frontoparietal SC and reasoning ability was present in particular in the RLPFC-IPL connection, for which the FC-reasoning relationship has been established. Further, we sought to determine whether there were differences between left- and right-side connections. Across the entire age range, there was a marginal positive relationship between RLPFC-IPL SC and reasoning (controlling for age and study: $b=19.1, t_{(128)}=1.6, p=$ 0.1 ). This result was driven by a significant effect in the left hemisphere (controlling for age and study: $b=23.1, t_{(129)}=2.0, p=$ $0.04)$, with no effect in the right hemisphere $(p=0.3)$. Consistent with what we had observed for average frontoparietal SC, we found a significant negative interaction between RLPFC-IPL SC and age $(b=-14.4, t=-4.9, p<0.001)$, as well as a significant main effect of SC on reasoning $\left(b=172.4, t_{(127)}=5.1, p<\right.$ $0.001)$. In this analysis, similar interaction effects were observed for left and right RLPFC-IPL connections (left: $b=-13.3, t_{(128)}=$ $-4.7, p<0.001$; right: $\left.b=-13.2, t_{(128)}=-5.8, p<0.001\right)$. Moreover, inclusion of the interaction term revealed a significant main effect of SC on reasoning for both the left and right RLPFC- 
IPL connections (left: $b=166.6, t_{(128)}=5.7$, $p<0.001$; right: $b=151.5, t_{(128)}=6.8, p<$ $0.001)$. Therefore, as for average frontoparietal SC, RLPFC-IPL SC demonstrated a positive association with reasoning ability that was stronger in younger than in older participants.

Finally, we sought to determine whether the relationship between SC and reasoning ability was mediated at least in part by FC. For this analysis, we focused on the left RLPFC-IPL connection that demonstrated the strongest relationship between FC and reasoning. Because the FC effect was limited to older participants and the SC effect was driven by younger participants, we did not expect to find a mediating relationship. Indeed, comparison of two models, one that included FC as a mediator of the SC-reasoning relationship and another that did not, revealed a clear preference for the model without mediation (Akaike information criterion: 4734 vs 3966, $p<0.001)$.

\section{Developmental changes in SC and FC}

To better understand the relationship between frontoparietal connectivity and reasoning, it is important to identify the developmental trajectory associated with each connectivity measure. We have previously reported a nonlinear pattern of age-related change for RLPFC-IPL FC, with the largest increases occurring during late childhood and early adolescence (Wendelken et al., 2016). Results from the current expanded sample are consistent with this finding: the optimal fit curve indicated a maximal rate of increase in FC at age $13\left(\mu=13, \sigma=2, b=0.15, t_{(1,583)}=\right.$ 10.8, $p<0.001$; Fig. $5 A)$.

For RLPFC-IPL SC, larger increases were observed in younger participants, with the maximal rate of increase at age $7(\mu=7$, $\sigma=6, b=0.07, t_{(1,778)}=14.7, p<0.001$; Fig. 5B). Average frontoparietal SC demonstrated a similar pattern of age-related change. In summary, developmental increases in RLPFC-IPL SC are greatest in younger children, whereas developmental increases in RLPFC-IPL FC peak in early adolescence.

\section{Relationships between SC and FC}

Having observed age-related and longitudinal increases in both structural and functional frontoparietal connectivity, we next sought to understand the relation between these two connectivity measures. Because both measures increased with age, it is unsurprising that they demonstrated a strong positive relationship, across our sample, before accounting for age $\left(b=1.6, t_{(206)}=5.6\right.$, $p<0.001)$. However, after accounting for age and study in a mixed-model regression, the relationship between average frontoparietal SC and average frontoparietal FC did not achieve statistical significance $\left(b=0.45, t_{(205)}=1.5, p=0.14\right)$. Further, there was no interaction with age $(p=0.8)$ or age group $(p=$ $0.6)$. Similar results were observed when the analysis was limited to the RLPFC-IPL connection (controlling for age and study: $\left.b=0.81, t_{(205)}=1.7, p=0.08\right)$. In this case, there were trendlevel positive interactions with age $(p=0.13)$ and with age group ( $p=0.17$ ). Therefore, we observed overall positive but weak concurrent relationships between frontoparietal SC and FC over and above the common effect of increasing age (Fig. 6).

Next, we conducted longitudinal analyses to test whether stronger SC at one time point might drive future increases in FC. We focused our investigation of such lead-lag dynamic relationships on the RLPFC-IPL connection. To test whether RLPFCIPL SC would predict future increases in corresponding FC, we conducted a linear stepwise regression with longitudinal change in FC as the dependent variable and $\mathrm{T} 1$ values for $\mathrm{FC}$, SC, and age and longitudinal change in age and $\mathrm{SC}$ as independent variables. The resulting model included T1 FC, T1 SC, T1 age, and change in SC as significant predictors of change in FC (Fig. 7A). As expected, T1 FC was a negative predictor of FC change $(b=-0.62$, $\left.t_{(134)}=-8.9, p<0.001\right)$ because individuals who already exhibit higher FC at T1 change less over time. Change in SC was a positive predictor of FC change, but this relationship was not significant after controlling for study $(p=0.4)$. Critically, T1 SC was a positive predictor of FC change (controlling for study; $b=1.7$, $\left.t_{(134)}=2.6, p=0.009\right)$, such that participants with higher RLPFC- 


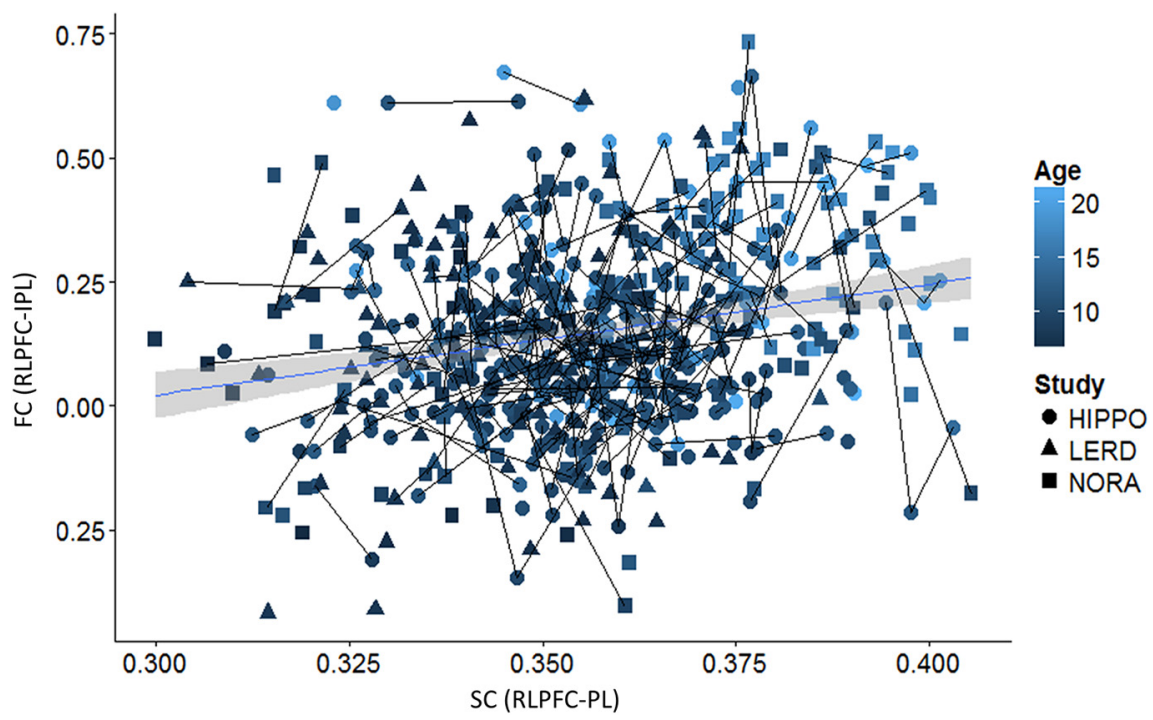

Figure 6. Scatter plot of the relationship between SC and FC for the RLPFC-IPL connection with a linear fit line. Lines between data points indicate longitudinal data.

A

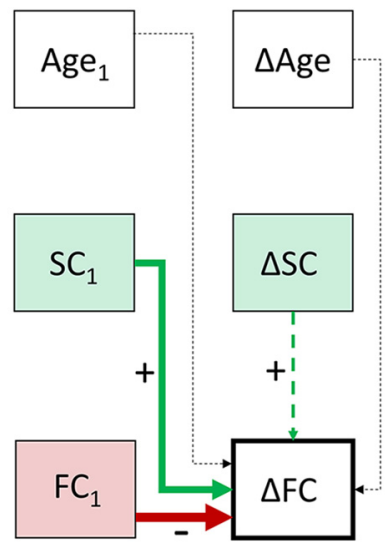

B

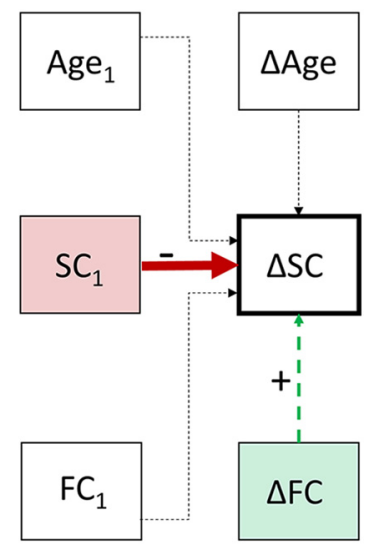

Figure 7. $\boldsymbol{A}$, Predictors of RLPFC-IPL FC change. $\boldsymbol{B}$, Predictors of RLPFC-IPL SC change. Shaded boxes indicate factors that survived stepwise regression. Solid lines indicate factors that survived both stepwise regression and correction for study site (with at least marginal significance).

IPL SC at T1 showed greater longitudinal increases in RLPFCIPL FC. There was no effect of age group on this result (T1 SC $\times$ group: $p=0.31$ ) nor was there an effect of gender (T1 SC $\times$ gender: $p=0.94)$. Similar effects of T1 SC on FC change were observed in each of the three datasets considered separately, although they only reached significance in the combined analysis (NORA: $b=1.5, p=0.18$; HIPPO: $b=1.6, p=0.07$; LERD: $b=3.3, p=0.16)$.

Having established that RLPFC-IPL SC predicts future changes in FC, we next sought to determine whether the converse relation was also present. To test whether RLPFC-IPL FC predicts future increases in corresponding SC, we conducted a linear stepwise regression analysis with longitudinal change in SC as the dependent variable and with $\mathrm{T} 1$ values for $\mathrm{FC}, \mathrm{SC}$, and age, and longitudinal change in age and $\mathrm{FC}$ as independent variables. The resulting model included $\mathrm{T} 1 \mathrm{FC}, \mathrm{T} 1 \mathrm{SC}, \mathrm{T} 1$ age, and FC change as predictors of SC change (Fig. 7B). After controlling for study, T1 SC was a negative predictor of SC change $\left(b=-1.8, t_{(135)}=\right.$ $-3.9, p<0.001)$, as expected. However, none of the other vari- ables (i.e., T1 age, T1 FC, and FC change) showed statistically significant values $(p>$ $0.39)$. In addition to a marginal negative relation between SC change and T1 age in the younger participants $(b=-0.001$, $p=0.08)$, a similar pattern of results was obtained for both age groups. Adding gender to the model yielded no additional effects. Further, examination of each of the three datasets separately revealed a similar lack of effect in each $(p>0.2)$. Therefore, we found no evidence of FC driving SC for the RLPFC-IPL connection.

\section{Connectivity and longitudinal changes in reasoning ability}

Finally, we sought to test whether structural and/or FC would predict longitudinal changes in reasoning ability. Because it was the left RLPFC-IPL FC that demonstrated the strongest cross-sectional association with reasoning ability, we narrowed our focus to this specific connection for all further analyses. We conducted a stepwise linear regression with change in reasoning as the dependent variable and T1 values for age, reasoning, SC for left RLPFC-IPL, and FC for left RLPFC-IPL and longitudinal changes in age, SC, and FC as independent variables. The resulting linear model included T1 reasoning, age, and SC.

As expected, $\mathrm{T} 1$ reasoning was a negative predictor of reasoning change (controlling for study: $b=-0.74, t_{(144)}=-11.1, p<$ $0.001)$. Both T1 SC and T1 age were positive predictors of reasoning change, though these effects were only marginally significant after controlling for study (SC: $b=$ 28.6, $t_{(144)}=1.8, p=0.07$; age: $b=0.24$, $\left.t_{(144)}=1.9, p=0.06\right)$. Notably, dropping either age or SC from the regression yielded a significant effect for the other factor (SC: $b=37.6, t_{(145)}=2.4, p=0.02$; age: $\left.b=0.31, t_{(145)}=2.6, p=0.01\right) . \mathrm{Nu}-$ merically similar but nonsignificant effects were observed separately in both datasets that contributed to this pooled analysis in NORA $(b=37.5, p=0.06)$ and in HIPPO $(b=38.0, p=0.16)$. Adding gender to this model yielded no additional effects or interactions.

Given the significant age effects that we had observed in our prior analyses relating SC and FC to reasoning ability, we repeated this analysis separately for younger and older participant groups even though we did not observe a significant interaction of T1 SC with either age or age group in the stepwise linear regression $(p>0.2)$. For younger participants, only T1 SC survived the stepwise regression as a positive predictor of reasoning change and it remained significant after controlling for age and study $\left(b=43.9, t_{(119)}=2.1\right.$, $p=0.04$; Fig. $8 A$ ). In contrast, for older participants, T1 age was the only positive predictor (age: $b=0.44, t_{(20)}=2.6, p=0.01$; Fig. $8 B$ ). $\mathrm{T} 1$ reasoning was a negative predictor in both groups, as for the entire sample. Therefore, the marginal relationship between left RLPFC-IPL SC and reasoning observed in the entire sample was driven by a significant positive relationship in children under age 12 . 


\section{Discussion}

To our knowledge, this study is the first to examine lead-lag relations between SC and FC within the frontoparietal network and the first to examine the dynamic leadlag relationship between these measures and reasoning ability. Below, we discuss the developmental changes observed for each of our measures separately and then discuss their dynamic interrelations.

\section{Age-related changes in SC, FC, and reasoning ability}

In all three of the measures that we examined, we found both cross-sectional agerelated increases as well as within-person longitudinal increases. However, distinct developmental trajectories were apparent. Reasoning ability exhibited large increases in younger children and relatively smaller but continuing increases into adulthood. This result is consistent with prior reports that have pointed to the early school years as a period of peak improvement in reasoning ability (McArdle et al., 2002; Ferrer et al., 2009).

In contrast, increases in frontoparietal FC were relatively minimal in younger children and were most pronounced at the point of transition between childhood and adolescence. This marked increase in frontoparietal FC in late childhood is consistent with reports from prior investigations (Smit et al., 2012; Barber et al., 2013; Ernst et al., 2015).

Frontoparietal SC demonstrated substantial increases in younger children and continuing change into adolescence. This demonstration of frontoparietal white matter development extended across a relatively broad age range is consistent with prior reports (Asato et al., 2010; Lebel et al., 2012). Notably, development of SC appears to both precede and follow the period of greatest change in FC.

\section{Frontoparietal FC supports reasoning in adolescents and adults}

As reported previously with the NORA dataset (Wendelken et al., 2016), we observed a positive concurrent relationship between RLPFC-IPL FC and reasoning ability in adolescents in the expanded sample. Among HIPPO study participants, who were not included in the previous report, this effect was marginally significant. Notably, the HIPPO sample did not include older adolescents, who made up a significant share of the NORA sample. Therefore, it is possible that the somewhat weaker effect for the HIPPO dataset was due to the different age distribution.

Although we and others have demonstrated the development of a concurrent relationship between frontoparietal FC and reasoning (Bazargani et al., 2014; Wendelken et al., 2016), we did not observe here any lead-lag developmental relationship between these two measures. Specifically, whereas RLPFC-IPL FC was associated with reasoning ability in adolescents and adults, the level of RLPFC-IPL FC did not predict future changes in reasoning ability. This outcome suggests that communication between RLPFC and IPL is characteristic of a mature reasoning system, but that robust communication between these regions may not be a prerequisite for the development of reasoning. In contrast, the maturation of frontoparietal white matter tracts during childhood may well be a prerequisite for the emergence in early ado-

\section{B Older participants (12-22 years)}

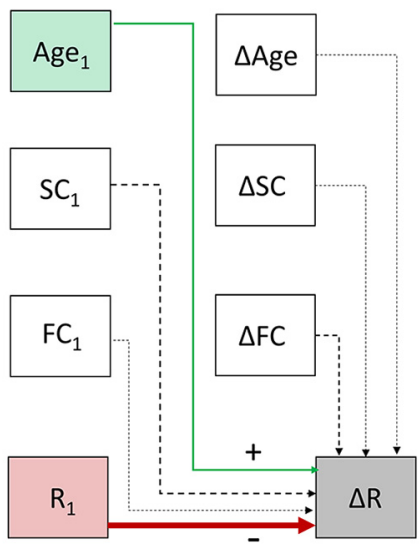

lescence of both advanced reasoning ability and the robust RLPFC-IPL FC on which this depends.

It is entirely possible that FC of different connections or involving other functional brain networks might predict future change in reasoning or other higher cognitive abilities. In addition to the frontoparietal network, the cingulo-opercular network, which is most frequently linked to cognitive control, has also been implicated in reasoning (Cocchi et al., 2014). Future investigations should assess whether cingulo-opercular and/or other connections also contribute to reasoning development.

\section{Frontoparietal SC supports the development of reasoning ability}

SC and FC had an impact on reasoning development. First, we observed a strong positive relationship between frontoparietal SC and reasoning ability in children. Although this relationship was not specific to the RLPFC-IPL connection, it does appear to be specific to frontoparietal connectivity and not to interhemispheric connections within the prefrontal or parietal cortices. This effect was not mediated by FC.

Second, we observed a lead-lag relationship between RLPFCIPL SC and reasoning ability in that higher SC led to subsequent increases in reasoning ability. Therefore, the state of white matter connection between RLPFC and IPL in childhood appears to be important for developmental improvements in reasoning ability.

Several prior studies have linked SC, and particularly frontoparietal SC, to reasoning ability and other higher cognitive operations. For example, Tamnes et al. (2010) reported a concurrent positive relationship between reasoning ability and FA within the cingulum bundle and inferior longitudinal fasciculus in children and young adults. Further, Peters et al. (2014) reported a concurrent association in children and young adults between FA in both cingulum bundle and inferior frontooccipital fasciculus and average performance across a range of cognitively demanding tasks. Using the NORA dataset that contributed to our current larger sample, we observed a positive relationship between global (average whole-brain) FA and reasoning ability in children and adolescents that was mediated by processing speed (Ferrer et al., 2013). Although we did not have a common measure of processing speed in our pooled dataset, we were able to extend our prior results by demonstrating a specific link between reasoning ability and frontoparietal connectivity, but not intrafrontal or intrapa- 
rietal connectivity, that we had not detected previously. These results build on our prior work by demonstrating that not all white matter pathways contribute equally to the development of reasoning.

\section{Frontoparietal SC supports emerging FC}

Because both SC and FC are seen to affect reasoning development, but at different points along the developmental trajectory, we considered it essential to our understanding of reasoning development to also understand the relationship between these two measures. For both the frontoparietal network generally, and more specifically for the RLPFC-IPL connection, SC and FC were not significantly related at a single time point after accounting for age and study site (despite a positive relation before accounting for these variables). This result is broadly consistent with prior investigations that have found overall positive concurrent relations between these measures but have also demonstrated FC in the absence of SC, consistent with the notion that FC reflects indirect (polysynaptic) as well direct (monosynaptic) communication between brain regions (Skudlarski et al., 2008; Honey et al., 2009).

Prior studies have told us something about the concurrent relationship between SC and FC, but they have not revealed the dynamic, lead-lag nature of this relationship. Here, focusing on the RLPFC-IPL connection, we found that SC was a predictor of future changes in FC. This result demonstrates that, at least for this connection, white matter connectivity enables the coordination between regions that is indexed by FC and, moreover, that strong white matter connectivity allows for the emergence over time of increased coordination between regions. On the grounds that increased coordination between regions would promote the development of white matter tracts via mechanisms of experiencedependent brain plasticity, we had hypothesized that robust FC should also lead to future increases in SC. However, at least for the RLPFC-IPL connection that was examined here, we found no evidence for this relationship.

\section{Limitations}

A virtue of the present study is the large number of subjects afforded by the combination of three separate and independent longitudinal datasets. However, this combination of datasets, which reused existing data collected for other purposes, is also the source of several important limitations. First, the fact that the data come from multiple sites with different research protocols and different scanning hardware means that nominally equivalent measurements may not be directly comparable. This problem was compounded by the fact that each dataset covered a different age distribution. We addressed this problem in two ways: first, we normalized data from the HIPPO and LERD datasets to age-matched samples from the NORA dataset; second, we included study as a covariate of no interest in relevant analyses. In additional to potential differences in nominally equivalent measures, there was even greater potential for differences across datasets in our measure of FC due to the fact that fMRI data came from different fMRI tasks or, in the case of LERD, from restingstate fMRI. Our FC data analysis, which involved low-pass filtering that excluded likely task frequencies, as well as explicitly regressing out task vectors, was designed to minimize these differences. Moreover, where the FC results demonstrate similar patterns in the separate datasets, this diversity may be seen as an asset. Nevertheless, the possibility that this difference between the datasets may have affected the FC results remains a key limitation of the current study. Finally, interpretation of relative effects in younger versus older participants is somewhat limited by the fact that the older group was considerably smaller that the younger group and, in some cases, by the fact that reasoning change was much greater among younger participants. In particular, either of these factors might contribute to the lack of relationship between $\mathrm{SC}$ and reasoning ability in older participants. It is important to note that the split at age 12 was motivated in part by the difference in reasoning trajectories and by previously observed differences in the FC-reasoning relationship (Wendelken et al., 2016). Therefore, we consider this to be the best choice for an age group split despite the size imbalance between the resulting subsamples. Analyses that incorporated age as a continuous variable, rather than categorical age groups, did not suffer from this limitation.

\section{Conclusion}

The present study is, to our knowledge, the first to examine the dynamic, lead-lag relationships between SC and FC and reasoning ability. We found that, whereas frontoparietal, and specifically RLPFC-IPL, FC is a key correlate of reasoning ability in adolescents and adults, it is the underlying frontoparietal SC that is more closely associated with reasoning ability in children both cross-sectionally and longitudinally. These results establish the importance of frontoparietal white matter development during childhood as a foundation for good cognitive functioning in adolescence. It will be important in future work to test the effects of demographic variables on the neurocognitive development of reasoning ability and also to determine whether childhood is a sensitive period for plasticity in the lateral frontoparietal network.

\section{References}

Asato MR, Terwilliger R, Woo J, Luna B (2010) White matter development in adolescence: a DTI study. Cereb Cortex 20:2122-2131. CrossRef Medline

Barber AD, Caffo BS, Pekar JJ, Mostofsky SH (2013) Developmental changes in within and between-network connectivity between late childhood and adulthood. Neuropsychologia 51:156-167. CrossRef Medline

Bazargani N, Hillebrandt H, Christoff K, Dumontheil I (2014) Developmental changes in effective connectivity associated with relational reasoning. Hum Brain Mapp 35:3262-3276. CrossRef Medline

Behrens TE, Woolrich MW, Jenkinson M, Johansen-Berg H, Nunes RG, Clare S, Matthews PM, Brady JM, Smith SM (2003) Characterization and propagation of uncertainty in diffusion-weighted MR imaging. Magn Reson Med 50:1077-1088. CrossRef Medline

Cocchi L, Halford GS, Zalesky A, Harding IH, Ramm BJ, Cutmore T, Shum DH, Mattingley JB (2014) Complexity in relational processing predicts changes in functional brain network dynamics. Cereb Cortex 24:22832296. CrossRef Medline

Cole MW, Bassett DS, Power JD, Braver TS, Petersen SE (2014) Intrinsic and task-evoked network architectures of the human brain. Neuron 83:238251. CrossRef Medline

Ernst M, Torrisi S, Balderston N, Grillon C, Hale EA (2015) fMRI functional connectivity applied to adolescent neurodevelopment. Annu Rev Clin Psychol 11:361-377. CrossRef Medline

Fair DA, Schlaggar BL, Cohen AL, Miezin FM, Dosenbach NU, Wenger KK, Fox MD, Snyder AZ, Raichle ME, Petersen SE (2007) A method for using blocked and event-related fMRI data to study "resting state" functional connectivity. Neuroimage 35:396-405. CrossRef Medline

Ferrer E, O'Hare E, Bunge S (2009) Fluid reasoning and the developing brain. Front Neurosci 3:46-51. CrossRef Medline

Ferrer E, Whitaker KJ, Steele JS, Green CT, Wendelken C, Bunge SA (2013) White matter maturation supports the development of reasoning ability through its influence on processing speed. Dev Sci 16:941-951. CrossRef Medline

Greicius MD, Supekar K, Menon V, Dougherty RF (2009) Resting-state functional connectivity reflects structural connectivity in the default mode network. Cereb Cortex 19:72-78. CrossRef Medline

Honey CJ, Sporns O, Cammoun L, Gigandet X, Thiran JP, Meuli R, Hagmann $P$ (2009) Predicting human resting-state functional connectivity from 
structural connectivity. Proc Natl Acad Sci U S A 106:2035-2040. CrossRef Medline

Horn A, Ostwald D, Reisert M, Blankenburg F (2014) The structuralfunctional connectome and the default mode network of the human brain. Neuroimage 102:142-151. CrossRef Medline

Jung RE, Haier RJ (2007) The Parieto-Frontal Integration Theory (P-FIT) of intelligence: converging neuroimaging evidence. Behav Brain Sci 30: 135-154; discussion 154-187. CrossRef Medline

Khalsa S, Mayhew SD, Chechlacz M, Bagary M, Bagshaw AP (2014) The structural and functional connectivity of the posterior cingulate cortex: comparison between deterministic and probabilistic tractography for the investigation of structure-function relationships. Neuroimage 102:118127. CrossRef Medline

Krawczyk DC (2012) The cognition and neuroscience of relational reasoning. Brain Res 1428:13-23. CrossRef Medline

Lebel C, Gee M, Camicioli R, Wieler M, Martin W, Beaulieu C (2012) Diffusion tensor imaging of white matter tract evolution over the lifespan. Neuroimage 60:340-352. CrossRef Medline

McArdle JJ, Ferrer-Caja E, Hamagami F, Woodcock RW (2002) Comparative longitudinal structural analyses of the growth and decline of multiple intellectual abilities over the life span. Dev Psychol 38:115-142. CrossRef Medline

Peters BD, Ikuta T, DeRosse P, John M, Burdick KE, Gruner P, Prendergast DM, Szeszko PR, Malhotra AK (2014) age-related differences in white matter tract microstructure are associated with cognitive performance from childhood to adulthood. Biol Psychiatry 75:248-256. CrossRef Medline

Pinheiro J, Bates D, DebRoy S, Sarkar D; R Development Core Team (2013) nlme: linear and nonlinear mixed effects models. $\mathrm{R}$ package version 3.1-109.

Power JD, Cohen AL, Nelson SM, Wig GS, Barnes KA, Church JA, Vogel AC, Laumann TO, Miezin FM, Schlaggar BL, Petersen SE (2011) Functional network organization of the human brain. Neuron 72:665-678. CrossRef Medline

Power JD, Barnes KA, Snyder AZ, Schlaggar BL, Petersen SE (2012) Spurious but systematic correlations in functional connectivity MRI networks arise from subject motion. Neuroimage 59:2142-2154. CrossRef Medline

Prado J, Chadha A, Booth JR (2011) The brain network for deductive reasoning: a quantitative meta-analysis of 28 neuroimaging studies. J Cogn Neurosci 23:3483-3497. CrossRef Medline

R Core Team (2013) R: A language and environment for statistical computing. Available from: http://www.R-project.org/. Accessed: January 1, 2016.

Sastre M 3rd, Wendelken C, Lee JK, Bunge SA, Ghetti S (2016) age and performance-related differences in hippocampal contributions to episodic memory retrieval. Dev Cogn Neurosci 19:42-50. CrossRef Medline Seeley WW, Menon V, Schatzberg AF, Keller J, Glover GH, Kenna H, Reiss
AL, Greicius MD (2007) Dissociable intrinsic connectivity networks for salience processing and executive control. J Neurosci 27:2349-2356. CrossRef Medline

Shaw P, Greenstein D, Lerch J, Clasen L, Lenroot R, Gogtay N, Evans A, Rapoport J, Giedd J (2006) Intellectual ability and cortical development in children and adolescents. Nature 440:676-679. CrossRef Medline

Shokri-Kojori E, Motes MA, Rypma B, Krawczyk DC (2012) The network architecture of cortical processing in visuo-spatial reasoning. Sci Rep 2:411. CrossRef Medline

Siegel JS, Mitra A, Laumann TO, Seitzman BA, Raichle M, Corbetta M, and Snyder A (2016) Data quality influences observed links between functional connectivity and behavior. Cereb Cortex. In press.

Skudlarski P, Jagannathan K, Calhoun VD, Hampson M, Skudlarska BA, Pearlson G (2008) Measuring brain connectivity: diffusion tensor imaging validates resting state temporal correlations. Neuroimage 43:554561. CrossRef Medline

Smit DJ, Boersma M, Schnack HG, Micheloyannis S, Boomsma DI, Hulshoff Pol HE, Stam CJ, de Geus EJ (2012) The brain matures with stronger functional connectivity and decreased randomness of its network. PLoS One 7:e36896. CrossRef Medline

Tamnes CK, Østby Y, Walhovd KB, Westlye LT, Due-Tønnessen P, Fjell AM (2010) Intellectual abilities and white matter microstructure in development: a diffusion tensor imaging study. Hum Brain Mapp 31:1609-1625. CrossRef Medline

Vendetti MS, Bunge SA (2014) Evolutionary and developmental changes in the lateral frontoparietal network: a little goes a long way for higher-level cognition. Neuron 84:906-917. CrossRef Medline

Wechsler D (1974) Manual for the Wechsler Intelligence Scale for Children-Revised. New York: The Psychological Corporation.

Wendelken C, Bunge SA (2010) Transitive inference: distinct contributions of rostrolateral prefrontal cortex and the hippocampus. J Cogn Neurosci 22:837-847. CrossRef Medline

Wendelken C, O’Hare ED, Whitaker KJ, Ferrer E, Bunge SA (2011) Increased functional selectivity over development in rostrolateral prefrontal cortex. J Neurosci 31:17260-17268. CrossRef Medline

Wendelken C, Chung D, and Bunge SA (2012) Rostrolateral prefrontal cortex: domain-general or domain-sensitive? Hum Brain Mapp 33:19521963. CrossRef Medline

Wendelken C, Ferrer E, Whitaker KJ, Bunge SA (2016) Fronto-parietal network reconfiguration supports the development of reasoning ability. Cereb Cortex 26:2178-2190. CrossRef Medline

Woodcock RW, McGrew KS, Mather N (2001) Woodcock Johnson III Tests of Achievement. Rolling Meadows, IL: Riverside.

Wright SB, Matlen BJ, Baym CL, Ferrer E, Bunge SA (2007) Neural correlates of fluid reasoning in children and adults. Front Hum Neurosci 1:8. CrossRef Medline 